\title{
Monitoring BReeding Bird Populations in Grand Teton National Park
}

\section{MARTIN L. CODY $\downarrow$ DEPARTMENT OF BIOLOGY UNIVERSITY OF CALIFORNIA $\downarrow$ LOS ANGELES}

\section{$\uparrow \quad$ GOALS}

The central aim of this project is to instigate a permanent program of monitoring landbird species composition and densities in a variety of representative habitats within Grand Teton National Park (GTNP). Habitats range from grassland and sagebrush on the valley floor of Jackson Hole (around $1900 \mathrm{~m}$ ) through a range of scrub, woodland, and tall foothill forest vegetation types to montane sites of subalpine fir and tundra (ca. $3000 \mathrm{~m}$ ). The monitoring program will provide data on year-to-year fluctuations in breeding bird species and densities, and eventually on any longer-term changes in the local avifauna, of both resident and migratory species; the data base will further understanding of population variability, local shifts in distribution and abundance, and potentially form a source for management and conservation decisions.

\section{$\uparrow \quad$ THE 1994 SEASON}

The 1994 season was an interim year, it followed a good deal of preliminary site selection and census activity (1966-1993) and is preliminary to full NPS funding of the project (scheduled to begin in 1995 with the collaboration of S. Cain, Natural Resources Manager, GTNP). In anticipation of range of habitats/sites eventually numbering 25-30, a) further site selection was undertaken, b) census methodology was refined, and c) new and prior sites were censused for breeding bird composition and density.
Table 1 lists 26 potential sites to be used in the monitoring study; 16 were established prior to 1994 (some as early as 1966-68), 7 new sites were initiated in 1994, and a further 3 were tentatively selected but not censused. The sites in Table 1 form. the basis of the permanent program, and will likely be augmented by several additional sites as need and opportunity arise (beginning with the 1995 season).

This year (1994) was particularly interesting from the standpoint of weather, being unusually dry and cool, with a number of potentially corresponding bird records of note. In particular, Clay-colored sparrow Spizella pallida was recorded on territory in the park, apparently for the first time since the early 1970 's, and a number of other rarities were present and breeding [including Bobolink Dolichonyx oryzivorus, Gray catbird Dumetella carolinensis, Lark sparrow Chondestes grammacus, and American redstart Setophaga ruticilla (the latter by S. Jones)].

\section{SITES AND CENSUS DATA}

Census data on 5 sites listed in Table 1 now span over a quarter century, and are already valuable in providing data on long-term population trends. Representative of such data are those of the Wet Willows site (\#9), as shown in Table 2. Eight species (listed first) have bred each census year since 1966; three are more sporadic, but have occurred throughout the census period; one species has not been recorded in the 1990's, and three others were not recorded in the earliest years of the censuses. Note that both parulid and emberizid guilds consist of 


\begin{tabular}{|c|c|c|c|c|}
\hline Site \# & Location & Habitat & $\operatorname{Elev}(\mathrm{m})$ & Yr Init. \\
\hline 1. & Wolf Ranch & Grazed meadow & 2045 & 1994 \\
\hline 2. & Jackson Lake Junction & Grass-sedge meadow & 2053 & 1992 \\
\hline 3. & Jackson Lake Junction & Grass-sagebrush & 2043 & 1966 \\
\hline 4. & Jackson Hole Airport & Tall sagebrush & 1956 & 1992 \\
\hline 5. & Two-ocean lake Rd. & Forb-meadow & 2158 & 1993 \\
\hline 6. & Elk Refuge & Wet grass-marsh & 1895 & 1994 \\
\hline 7. & Shadow Mt. & Aspen-scrub & 2090 & 1994 \\
\hline 8. & RKO/Snake River Bottoms & Dry willow flats & 2003 & 1993 \\
\hline 9. & Jackson Hole Junction & Wet willow flats & 2049 & 1966 \\
\hline 10. & Oxbow bend & Willow-aspen & 2040 & 1992 \\
\hline 11. & Elk Ranch West & Low aspen woodland & 2109 & 1968 \\
\hline 12. & Elk Ranch East & Mid aspen woodland & 2152 & 1968 \\
\hline 13. & Signal Mountain & Tall aspen woodland & 2091 & 1968 \\
\hline 14. & Spread Creek & Cottonwoods & 2085 & 1992 \\
\hline 15. & Schwabacher Landing & Cottonwoods & 1988 & 1992 \\
\hline 16. & Beaver Creek* & Lodgepole (1985 burn) & 2085 & \\
\hline 17. & Granite Canyon mouth* & Lodgepole-aspen wdland & 1965 & \\
\hline 18. & Timbered Island Moraine & Lodgepole-spruce & 2060 & 1994 \\
\hline 19. & AMK Ranch & Lodgepole pine forest & 2055 & 1991 \\
\hline 20 . & Signal Mountain & Lodgepole-fir forest & 2258 & 1992 \\
\hline 21. & Lizard Creek & Lodgepole-spruce-fir & 2194 & 1991 \\
\hline 22. & Jenny Lake & Spruce-fir forest & 2197 & 1992 \\
\hline 23. & Bradley Lake* & Spruce-fir forest & 2180 & \\
\hline 24. & Upper Granite Canyon & Spruce forest & 2365 & 1994 \\
\hline 25 . & Rendezvous Mountain & Subalp. fir-limber pine & 2970 & 1994 \\
\hline 26. & Cody Bowl & Arctic-alpine tundra & 2900 & 1994 \\
\hline
\end{tabular}

*Proposed sites not yet initiated.

Table 2. Breeding bird community in the Wet Willows site (\#9, Table 1), showing breeding density in units of prs/ha.

\begin{tabular}{|lllllll|}
\hline Year of census: & 1966 & 1991 & 1992 & 1993 & 1994 & CV \\
\hline Yellow warbler & 1.99 & 2.00 & 2.61 & 2.91 & 2.67 & 0.17 \\
Wilson's warbler & 1.81 & 1.55 & 0.40 & 1.10 & 0.64 & 0.54 \\
Northern yellowthroat & 3.08 & 1.45 & 2.06 & 2.55 & 2.50 & 0.26 \\
Willow flycatcher & 0.36 & 0.50 & 0.37 & 0.41 & 0.49 & 0.15 \\
Lincoln's sparrow & 1.99 & 1.27 & 0.92 & 0.89 & 1.05 & 0.37 \\
Fox sparrow & 1.00 & 0.82 & 1.04 & 1.21 & 1.01 & 0.14 \\
Song sparrow & 0.90 & 1.36 & 1.44 & 1.72 & 1.55 & 0.22 \\
Wh.-crowned sparrow & 0.36 & 0.82 & 0.64 & 0.86 & 0.77 & 0.29 \\
Calliope hummingbird & 1.36 & -- & 0.09 & 0.07 & 0.58 & 1.37 \\
MacGillivray's warbler & 0.09 & -- & -- & 0.26 & 0.08 & -- \\
Swainson's thrush & 0.27 & -- & 0.03 & -- & -- & 0.15 \\
Clay-colored sparrow & 0.72 & -- & -- & -- & 0.05 & 0.22 \\
Northern waterthrush & -- & -- & -- & 0.06 & 0.20 & -
\end{tabular}

Summary totals for above 15 mapped species:

\begin{tabular}{lllllll} 
\# Species breeding: & 12 & 8 & 13 & 11 & 13 \\
Tot. breeding density: & 14.93 & 9.77 & 10.09 & 12.18 & 11.76 & 0.18 \\
& & 4 & 3 & 4 & 4 & 5 \\
\# Paruline spp.: & 4.97 & 5.00 & 5.25 & 6.82 & 6.04 & 0.15 \\
Tot. breeding density: & 5 & 4 & 5 & 5 & 5 & \\
\# Emberizine spp.: & 5.97 & 4.27 & 4.10 & 4.88 & 4.43 & 0.16 \\
Tot. breeding density: & & & & & & \\
\hline
\end{tabular}


species with a range of CV (coefficient of variation of inter-year density) values with a 2-3 fold range, whereas the summed densities within guilds show far more modest variation, and support the notion of intraguild density compensation. The site also provides support for the influence of a) habitat change within site, and b) long-term change in population levels extrinsic of site characteristics (Cody, 1996 in press).

A feature of census replicability in GTNP is the influence of habitat type. Woodland and forest habitats yield consistent results among years, whereas meadow habitats are far more variable. For example, lodgepole pine forest (AMK Ranch, site \#19) supported 21-27 species over three years (Table 3 ), and about $80 \%$ of the passerine species listed are present in each year; total bird density varied by $46 \%$, and pairs/species between 1.75 and 2.0 . In contrast, meadow habitat (e.g. Two Ocean Lake meadow, site \#5) varied two-fold in both species count and total density in adjacent years, 19931994; this site is remarkable in supporting some 11 species of finches and emberizine (Table 4).

\begin{tabular}{|c|c|c|c|c|c|c|c|c|c|c|}
\hline AREA: 10 ha DT/hr: & & & Summ & 1015 & 600 & Summ & 1500 & 1730 & 600 & Summ \\
\hline SPECIES & 62092 & 62692 & 1992 & 62493 & 70293 & 1993 & 62594 & 62994 & 70394 & 1994 \\
\hline Br. head. cowbird & 1.5 & 2 & 2 & 1.5 & & 1.5 & & & 1 & 1 \\
\hline Raven & 0.5 & 0.5 & 0.5 & 0.5 & 0.5 & 0.5 & 0.5 & 0.5 & 0.5 & 0.5 \\
\hline Wh. cr. sparrow & & 1 & 1 & 1 & 0.5 & 1 & & & 0.5 & 0.5 \\
\hline Robin & 1.5 & 4 & 3 & 2 & 3 & 3 & & 1 & 1 & 1 \\
\hline Red-sh. flicker & 1 & 1 & 1 & 1.5 & 1.5 & 1.5 & & 1 & 1 & 1 \\
\hline Red-tailed hawk & & & & & & & 0.2 & 0 & & 0.1 \\
\hline Br. tail. hummingbird & & & & & & & & 0.5 & & 0.5 \\
\hline Violet-gr. swallow & 1 & & 0.5 & & & & & 1 & & 1 \\
\hline Warbling vireo & 2 & 1.5 & 2 & 2 & 2 & 2 & 2.5 & & 2.5 & 2.5 \\
\hline Swainson's thrush & 1 & 1 & 1 & 1 & & 1 & & & 1 & 1 \\
\hline Ddusky flycatcher & & & & & & & & & 1 & 1 \\
\hline Red-n. sapsucker & 1 & & 0.5 & & & & & & & \\
\hline W. wood pewee & 4 & 4 & 4 & 1 & 2 & 2 & & 2 & & 2 \\
\hline Yellow-r. warbler & 4 & 3.5 & 4 & 4.5 & 3 & 3.5 & 2 & 1 & 6.5 & 6 \\
\hline Dark-eyed junco & 5 & 9 & 8 & 6 & 3 & 5 & 2 & 3.5 & 6 & 5 \\
\hline Pine siskin & 2 & 2 & 2 & 1 & 1 & 1 & 4 & 4 & 3 & 4 \\
\hline Chipping sparrow & 2 & 5 & 4 & 0.5 & 3 & 3 & & 2 & 4 & 4 \\
\hline Red crossbill & 1 & 1 & 1 & & & & & & 2 & 1.5 \\
\hline Ruby-cr. kinglet & 3 & 6 & 5 & 3 & 3.5 & 3.5 & 2 & 2 & 2 & 2 \\
\hline Cassin's finch & 2 & 1 & 2 & 1 & 1 & 1 & & 2 & 2 & 2 \\
\hline Pine grosbeak & & & & & 1 & 0.5 & & & 1 & 0.5 \\
\hline Red-br. nuthatch & 3 & 3 & 3 & 2 & 1 & 2 & & 1 & 3 & 3 \\
\hline Mt. chickadee & 3 & 2 & 3 & 1 & 1 & 1 & 1 & 2 & 2 & 2 \\
\hline Gray jay & 1 & 0.25 & 0.5 & 1 & & 0.5 & & & 1 & 0.5 \\
\hline Clark's nutcracker & 1 & 0.5 & 0.75 & & & & & & 0.5 & 0.5 \\
\hline Western tanager & 1 & 2.75 & 2.5 & & 2 & 2 & 2 & 1 & 2 & 2 \\
\hline Ol. sided flycatcher & 1 & 1 & 1 & & & & & & & \\
\hline Hairy woodpecker & 1 & 1 & 1 & 1 & 1 & 1 & & & 1 & 1 \\
\hline Blue grouse & & 1 & 0.5 & & & & & & & \\
\hline Brown creeper & & 1 & 0.5 & & & & & & & \\
\hline Hermit thrush & & & & & 1 & 0.5 & & & & \\
\hline & & & & & & & & & & \\
\hline & & & & & & & & & & \\
\hline & & & & & & & & & & \\
\hline TOT. SPP.: & 23 & 24 & 26 & 18 & 18 & 21 & 11 & 16 & 22 & 27 \\
\hline TOT. DENS.: & 43.5 & 55 & 54.25 & 30.5 & 31 & 37 & 19.7 & 24.5 & 46 & 48.1 \\
\hline
\end{tabular}


Table 4. Bird census results from Two Ocean Lk Meadow, Site \#5, 1993-1994.

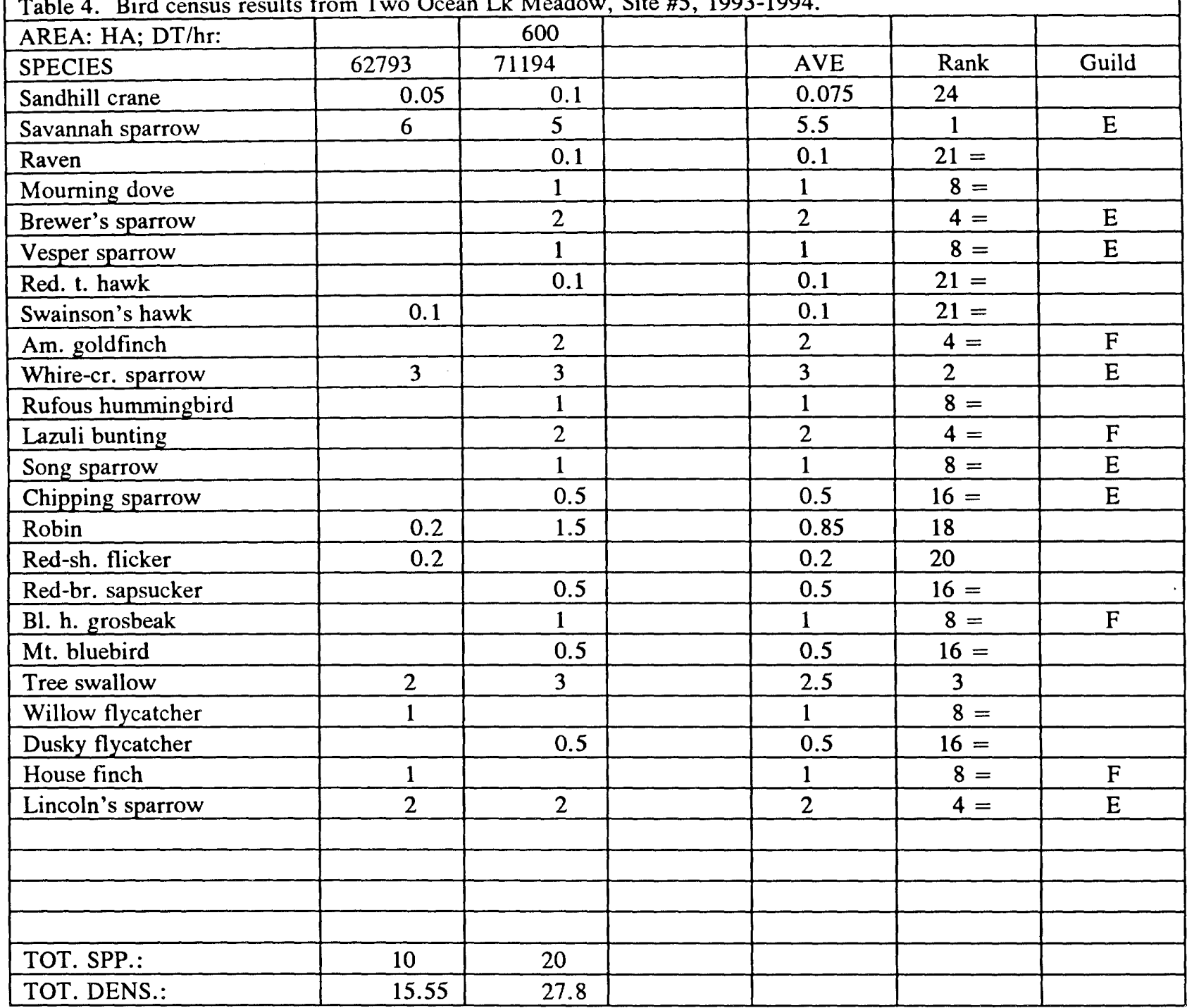

\section{$\downarrow \quad$ PROJECTED 1995 SEASON}

The monitoring project will move to fully active status in the 1995 season, when the remaining census sites will be initiated and all sites censused on a formal rotation with an established protocol. Sites will be GPS-established with permanent markers, and habitat/vegetation measurements conducted.

\section{LITERATURE CITED}

Cody, M.L. 1996. Bird communities of the Central Rocky Mountains. Ch. XX in Long-term Studies of Vertebrate Communities, eds. M.L. Cody and J.A. Smallwood, Academic Press, Orlando (in prep.). 